\title{
Development of Optical Wireless Audio System Using Infrared Light Communications
}

\author{
N. R. Mohamad ${ }^{1}$, A. A. Wakiran ${ }^{2}$, M. F. Iswandi ${ }^{3}$, A. Salleh ${ }^{4}$, \\ S. A. M. J. Yunus ${ }^{5}$, F. Sallehuddin ${ }^{6}$, N. F. Azmi ${ }^{7}$, S. A. M. Chachuli ${ }^{8}$ \\ 1,2,3,4,5,6,7,8 Center for Telecommunication Research \& Innovation (CeTRI), Faculty of Electronic \& Computer \\ Engineering, Universiti Teknikal Malaysia Melaka, Hang Tuah Jaya, 76100 Durian Tunggal, Melaka, Malaysia
}

\begin{abstract}
A simple prototype of an optical wireless audio system by using Infrared (IR) as a source for the transmitter has been proposed. In this project, we designed and implement a wireless optical transmitter and receiver system that established an audio communication at some distances with weak signal via infrared communication. In addition, this project also proposed infrared communication at certain angle which is a new solution or techniques to undertaken the characteristic of light that propagates in a straight line although the signal is very low or weak over long distance. The wavelength that used for this project is 870nm which is infrared wavelength range. Based on the experimental results, the infrared communication system can work successfully within in distance up to 10 meters and sensitivity angle of the photodiode at receiver that view the infrared light coming from the transmitter is up to $140^{\circ}$. This project is successfully improved the transmission distance and signal coverage area effectively with low cost of hardware, high communication speed and almost no limit to bandwidth.
\end{abstract}

Keywords: Optical wireless communication, free space optical, viewing angle, infrared, audio transmission

\section{INTRODUCTION}

The Optical Wireless Communication (OWC) also known as Free Space Optical (FSO) system is a system that modulates visible or infrared (IR) beams through the atmosphere to propagate any data signals in a system that transfer a signal via visible light usually the wavelength is $380 \mathrm{~nm}$ to $740 \mathrm{~nm}$ through free space [1]. In other words, it uses light to propagate the signal or to transmit the data in free space. FSO system uses light as the optical source such as a light emitting diode (LED) or laser diodes (LD) to transmit a data instead of enclosing the data stream in a glass fiber but it transmits the modulated light beam through the air. Hence, OWC is gaining reception in an increasing to the number of sectors of science and industry, owing to is a very unique combination of the features such as extremely high bandwidth, rapid deployment time, license and tariff-free bandwidth allocation, low power consumption, weight and size [1].

The system uses infrared technology has become a significant advantage over a radio frequency (RF). Therefore, optical wireless IR knowledge seems to be perfect for managing wireless communication in the future [2]. Furthermore, infrared transmission does not interfere with the existing RF system and not controlled by the Federal Communication Commission (FCC) regulations. In addition, this project can build some privacy for sending and receiving data as infrared does not penetrate the wall, thus providing a level of privacy in the area [3].

In 2005, R. Kawano et al. has reported the technology of compactness with high-quality sound generation. The digital signal is converted by ON-OFF keying and transmitted via an infrared LED with 1-bit $\mathrm{A} / \mathrm{D}$ conversion. The receiver converts the received infrared signal into an electrical signal and then the logic inverter reshapes the waveforms and converts them to analog signal with a simple low pass filter. Finally, the analog signal drives the speaker to regenerate the voice. This simple receiver configuration makes the receiver very small and inexpensive [4]. However, the transmission distance is only 3 meters to 5 meter and its depends upon the number of the transmitter. Besides that, A. Beaver et al. also reported that the distances of operation vary with the intensity of the infrared light that is emitted by the LED on the transmitter. The number of LED that emitting light is controlled by a single jumper. Each jumper configuration limits the current through the LED to approximately $70 \mathrm{~mA}$, where the maximum current the LED can take is $100 \mathrm{~mA} \mathrm{[5].} \mathrm{Wenqi} \mathrm{Huang} \mathrm{and}$ Hong Yang have reported that the method modulation and demodulation are the key technology of the transmitting terminal and receiving terminal respectively. The scheme I (Laser scheme) use the semiconductor visible laser with wavelength is $650 \mathrm{~nm}$ as the signal carrier and a photoelectric diode as the detector, the scheme II (Infrared scheme) uses infrared as the signal carrier and an infrared remote control receiver module as the detector, the scheme III (Radio scheme) use radio wave as the signal carrier and a receiving antenna as the detector [6]. The scheme infrared transmission distance is 5 meters, and some small obstacles are allowed 
because the infrared's transmission path is divergent. Nevertheless, the background noise and vibration environment will also influence the effect of receiving.

Furthermore, the newest publications have attracted considerable interest in using wireless optical communication links in aircraft and space vehicles. Wide Light Emitting Diode (WLED) has been widely used in lighting for the cabin in the new generation of commercial aircraft. Using on-board wireless optical communication has great potential for size, weight, power, cost and EMI reduction [7]. Therefore, this project will provide the initial drive to transmit voice and music by the light source and propagated through free space to be received with improving the distance up to 10 meters and more value for viewing angle. Moreover, this project also undertaken as a solution for problems about limited distance for transmitting the audio signal and reduce the noise.

\section{Circuit Design}

Fig. 1 shows the basic diagram of the optical wireless audio system or free space optical system (FSO) where the system are called line of sight (LSO) simplex communication system. The light from transmitter must be transmitted in straight lines without any divergence or scattering. It is divided into two main parts consist of the transmitter circuit and a receiver circuit as shown in Fig. 2 and Fig. 3 respectively. For this project, the circuit for transmitter and receiver has being designed by using Proteus software and the circuit is fabricated using printed circuit board (PCB) as shown in Fig. 4.

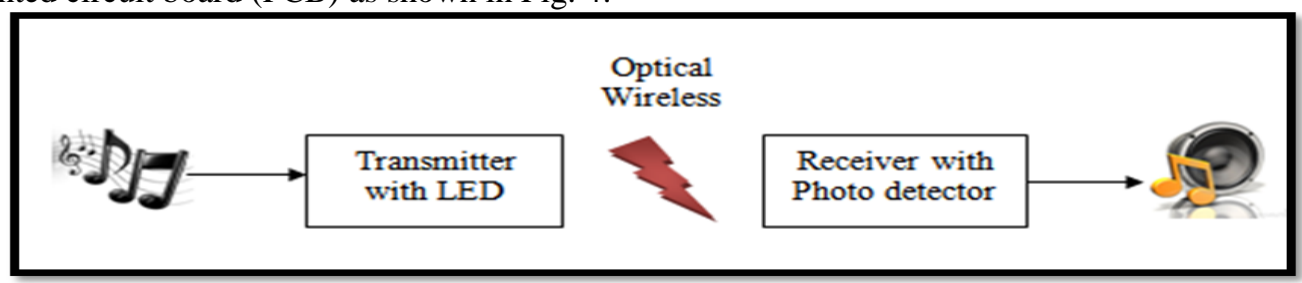

Fig. 1: Basic diagram of the optical wireless audio system

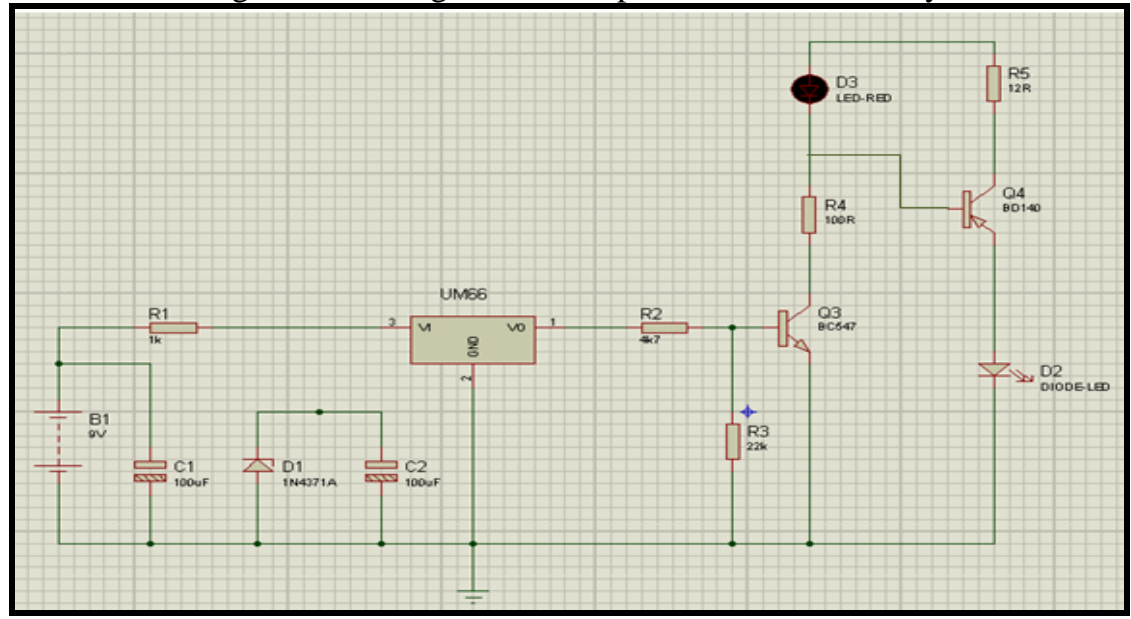

Fig. 2 : Designing of transmitter circuit by using Proteus software

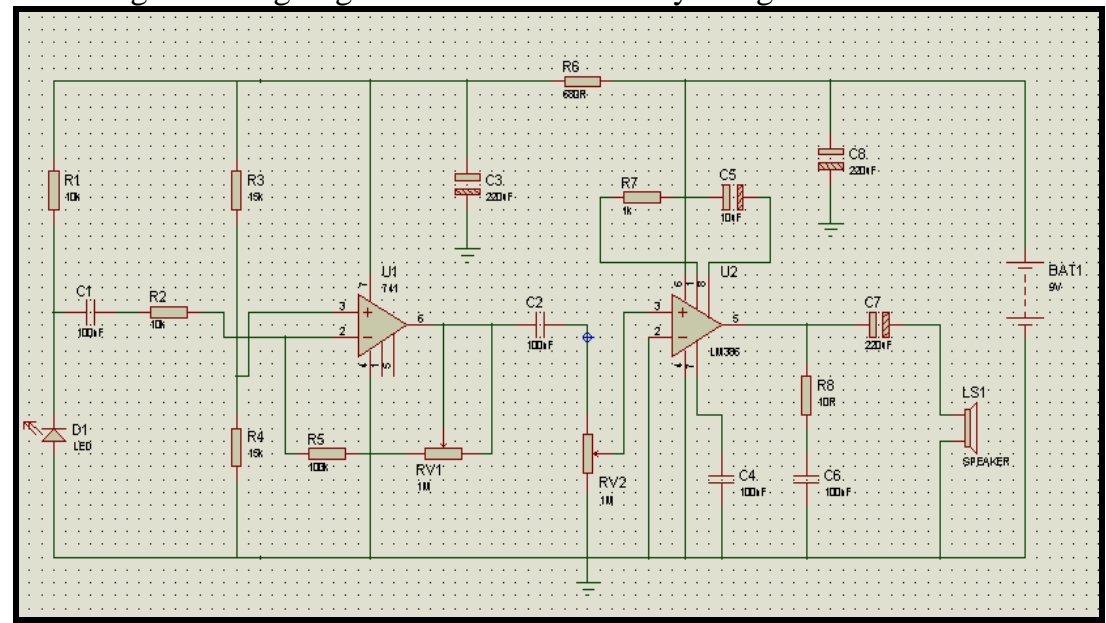

Fig. 3 : Designing of receiver circuit by using Proteus software 


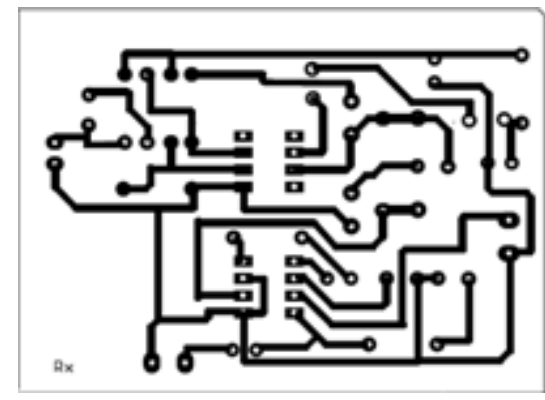

a) Transmitter circuit

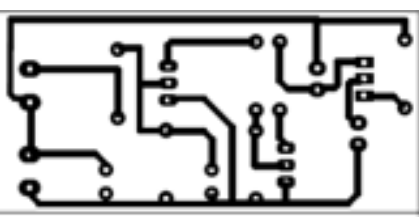

b) Receiver circuit

Fig. 4 : PCB layout

\section{Circuit Operation}

Infrared (IR) audio transmitter system works off $9 \mathrm{~V}$ battery. Fig. 2 shows the transmitter circuit by using infrared (IR) emitter LED as the light sources. It uses popular melody generator IC UM66 that can generate a continuous musical tone. UM66 IC production is fed to the infrared (IR) driver stage (built cross transistors BC547 and BD140) to get the maximum range. Here, the red LED flashes according to musical tones generated by UM66 IC, indicating modulation. The electrical signal from melody generator UM66 is converted into an invisible infrared light signal by the infrared light emitting diode (IR LED). For maximum sound transmission needs to be oriented towards and must hit the infrared (IR) photodiode at the receiver. In addition, to transmit the light over a longer distance, a brighter IR LED is needed or an invisible light has to be focused using lens. The photodiode in the receiver converts this invisible infrared light signal into an electrical signal at the receiver circuit as shown in Fig. 3. The infrared (IR) audio system receiver uses popular operational amplifier IC741 and audio frequency amplifier IC LM386 along with photodiode and some discrete components. The melody generated by IC UM66 is transmitted through IR LED, received by photodiode and fed to pin 2 of IC741. Its gain can be varied using variable resistor VR $\mathrm{VR}_{1}$. The output of IC741 is fed to IC LM386 via capacitor and variable resistor $\mathrm{VR}_{2}$. ). Finally, this larger electrical signal drives the speaker which turns electrical energy into sound energy. The melody produced is heard through the receiver loudspeaker. Variable resistor $\mathrm{VR}_{2}$ is used to control the volume of loudspeaker $(8 \Omega, 1$ WThe volume of melody can control with $1 \mathrm{M}$ variable resistor that connected to LM386.

\section{RESUlTS AND Discussions}

Fig. 5 shows the completed prototype of optical wireless audio system. Both IR transmitter and receiver work by using battery (9V). IR LED is infrared transmitting LEDs. Transmitter uses light as a data carrier. Transmitter was modulation frequency to produce a series of audio signal encoding. Then, the receiver will receive the signal light, modulated and enforced signals that can be sent by voice amplifier where the music can be heard.

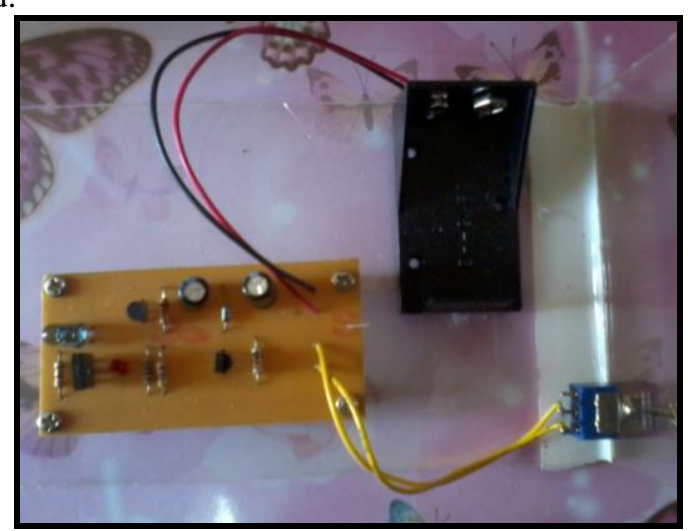

a) Transmitter circuit

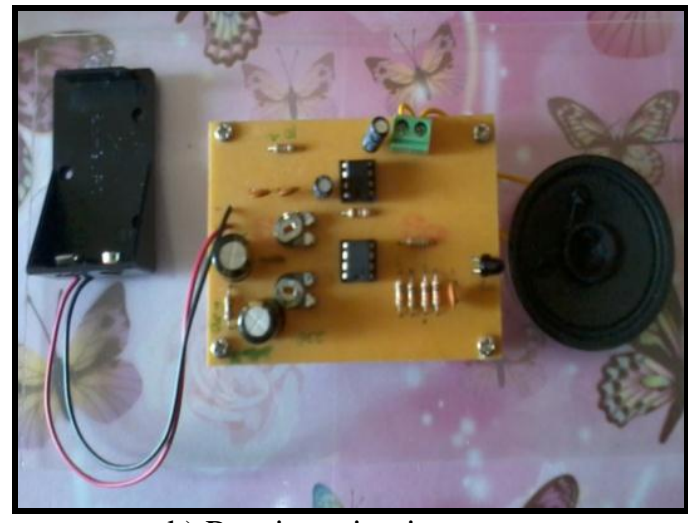

b) Receiver circuit

Fig. 5 : The constructed prototype of the transmitter and receiver

Table 1 and Fig. 6 show the data analysis between the voltage values of photodiode when the distances increase. From the analysis, the voltage will be decrease proportionally with the distance from 1 meter until 10meter. Therefore, over long distances, the photodiode voltage drop and produce low output (low melody). 
Table 1: Voltage of the photodetector versus distance

\begin{tabular}{|c|c|}
\hline Vout (Photodetector) & Distance (meter) \\
\hline $643 \mathrm{mV}$ & 1 \\
\hline $585 \mathrm{mV}$ & 2 \\
\hline $440 \mathrm{mV}$ & 3 \\
\hline $367 \mathrm{mV}$ & 4 \\
\hline $227 \mathrm{mV}$ & 5 \\
\hline $169 \mathrm{mV}$ & 6 \\
\hline $88 \mathrm{mV}$ & 7 \\
\hline $86 \mathrm{mV}$ & 8 \\
\hline $84 \mathrm{mV}$ & 9 \\
\hline $80 \mathrm{mV}$ & 10 \\
\hline
\end{tabular}

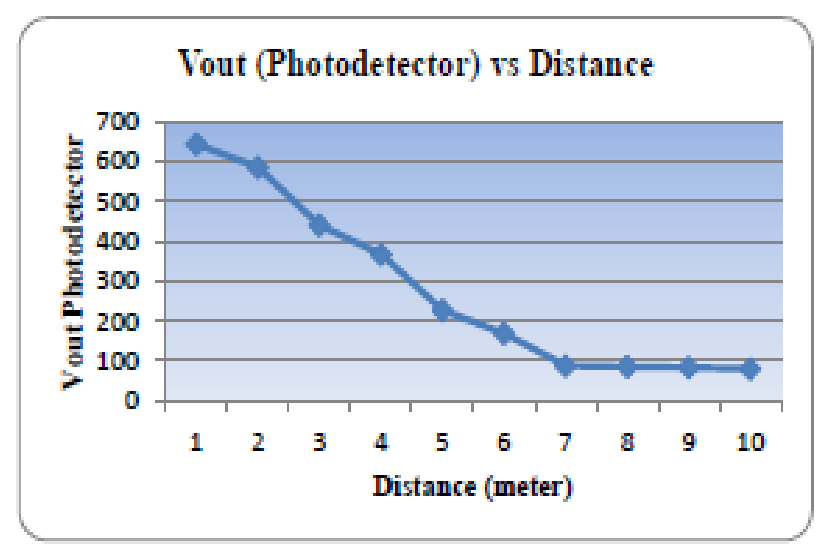

Fig. 6 : Voltage of the photodetector versus distance

Table 2 shows the measurement of viewing angle and the value of voltage at the photodiode. From the observation, increase the degree from transmitter circuit and receiver circuit, the value of voltage was decrease. The result with output waveform was shown in Table 2: Viewing angle versus distance has been measures using oscilloscope [8].

Table 2: Viewing angle versus distance

\begin{tabular}{|l|l|l|l|}
\hline Degree & \multicolumn{2}{|c|}{ Output Waveform } & Voltage value (mV) \\
\hline $90^{\circ}$ & & & \\
\hline $120^{\circ}$ & & & \\
\hline $140^{\circ}$ & & & \\
\hline & & & \\
\hline
\end{tabular}




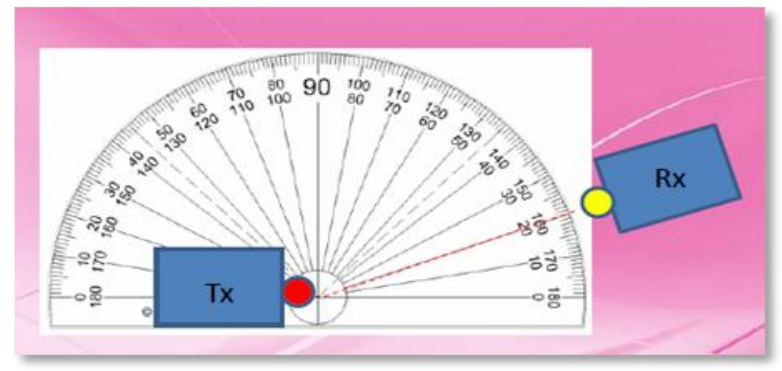

Fig. 7: Viewing Angle, Tx is short form for transmitter and Rx is receiver.

\section{CONCLUSION}

The optical wireless audio system can be used to transmit musical information from the receiver end to the transmitter end using the infrared radiations. Based on this project, we have improve the traditional infrared communication by increasing the transmission distance and the effective signal coverage area, which is also has the advantage of low cost, high speed communication and almost no bandwidth limits. The circuit can use for the wireless communication in the range of 7-10 meters and viewing angle of the Infrared (IR) LED radiation is up to $140^{\circ}$. In the future, we recommend to improve the efficiency of optical wireless transmission by using laser diodes (LD) as the light source, because the LD can hit the laser over long distance with low noise. Furthermore, this project will proceed to transmit audio and video simultaneously with low noise in the future. Therefore, this system should be a good system and has high potential of commercialization that can be used in various applications such as wireless communication in the museum, airplane, intercom, TV sound system and closed circuit cameras.

\section{ACKNOWLEDGEMENTS}

We would like to thank Universiti Teknikal Malaysia Melaka (UTeM) which has financially supported this research to be accomplished.

\section{REFERENCES}

[1] H. Manor and S. Arnon, Performance of an optical wireless communication system as a function of wavelength, Appl. Opt. 42, 2003, 4285-4294.

[2] J. M. Kahn and J.R.Barry, Wireless Infrared Communications, Proceedings of the IEEE, vol. 85, no. 2, February 1997, 265-298.

[3] A. C. Boucouvalas, Indoor Ambient Light Noise and its Effect on Wireless Optical Links, IEEE Proc. Optoelectronics, vol. 143, no. 6, Dec. 1996, 334-338.

[4] R. Kawano, Y. Matsuya, T. Ishihara, J-I. Inoue, and Y. Kado, Voice Ubique Ultra Small High Quality Infrared Audio Receiver, Proc. IEEE Int. Conf. Consumer Electron, 2005, 285 -286

[5] A. Beaver, M. Hannon, and A. Jalilzadeh, Infrared FM Audio Communication System, 2011.

[6] W. Huang and H. Yang, Comparative study on three methods of wireless receiving of audio signal, 2nd Inter. Conf. on. Artificial Intelligence, Management Science and Electronic Commerce (AIMSEC), 2011, 1994-1997.

[7] E. Chan, Wireless Optical Links for Airplane Applications, IEEE, Photonics Society Summer Topical Meeting Series, 2012, 76-77.

[8] Najmiah Radiah Mohamad, Chau Man Siang, Ahmad Sadhiqin Mohd Isara, Adie Mohd Khafe, Azahari Salleh, Nur Fatihah Azmi, Electromagnetic Levitator, International Conference on Computer and Communication Engineering(ICCCE), 2012, 319-324. 